\title{
ON THE COMPATIBILITY OF A LOGARITHMIC TURBULENT BOUNDARY LAYER VELOCITY PROFILE WITH EXPERIMENTAL DATA
}

\author{
A. ZARYANKIN, A. ROGALEV, V. KINDRA, G. KURDIUKOVA \& A. VEGERA \\ Moscow Power Engineering Institute, Russia.
}

\begin{abstract}
The compatibility of the semiempirical turbulence theory of L. Prandtl with the actual flow pattern in a turbulent boundary layer is considered in this article, and, based on this theory, the final calculation results of the boundary layer are analyzed. These show that the accepted additional conditions and relationships, which integrate the differential equation of $\mathrm{L}$. Prandtl, associating the turbulent stresses in the boundary layer with the transverse velocity gradient, are fulfilled only in the near-wall region, where the mentioned equation loses meaning, and are inconsistent with the physical meaning in the main part of integration.

It is noted that an introduced concept regarding the presence of a laminar sublayer between the wall and the turbulent boundary layer is the way to give a physical meaning to the logarithmic velocity profile.

It shows that coincidence of the experimental data with the actual logarithmic profile is obtained as a result of the use not of a particular physical value, as an argument, but of a function of this value. In this way, the coincident experimental points in general are concerned with different sections of the boundary layer. Accordingly, the informational value of the comparison of the calculations and the experiment given in the literature is actually eliminated.

Keywords: boundary layer, dynamic velocity, 'floating' coordinates, laminar sublayer, turbulence.
\end{abstract}

\section{INTRODUCTION}

The fact that all current programs of gas-dynamic calculations are based on the Reynolds equations, describing turbulent flow motions in general, has enabled the continuous calculation of the velocity and pressure fields in real fluids and gases without their division into two regions: the boundary layer regions, where the impact of viscous turbulent stresses is concentrated and the outer region, where the effect of tangential stresses can be neglected.

In this case, the central part of classical theoretical fluid and gas dynamics - a boundary layer theory determining the physics of moving fluids and gases with streamlined surfaces is excluded.

However, such a radical conclusion is justified only where there is a guarantee that a theoretical base, which forms the modern programs of the gas-dynamic calculations, is unquestionable.

Unfortunately, in the present case, this is far from true as in 1924 A.V. Keller and A.A. Friedman showed the impossibility of correctly closing the Reynolds equations [1].

Some later works [2, 3] contain similar statements. Reflections discussed in these works remain topical and give a full scientific assessment to that theoretical base, which is fundamental for modern programs of fluid-dynamics calculations using several phenomenological turbulence theories to close differential equations of turbulent motion.

In a methodological plan, each such theory is tested on a certain type of task. Accordingly, when passing to another type of task solution, the closing relationships shall be corrected again, using another turbulence theory. 
For the first time, a similar solution methodology of the unclosed differential equation was used to find the velocity profile in the turbulent boundary layer based on the Prandtl semiempirical turbulence theory.

The results of this classical solution provide the basis for the formation of flow physics in the turbulent near-wall layer.

Let us examine in detail the extent to which the tasks, assumptions, statements and hypotheses introduced in solving the above-mentioned task correspond to the facts, and how correctly the comparison of calculation and experimental data is implemented.

\section{THE LOGARITHMIC VELOCITY PROFILE IN THE TURBULENT BOUNDARY LAYER}

The solution to the above-mentioned task in its classic case is based on the known Prandtl relation, determining the connection of the turbulent shear stress with the averaged transverse velocity gradient in the boundary layer:

where, $\tau$ - turbulent stress $(\mathrm{Pa})$,

$$
\tau=\rho l^{2}\left(\frac{d u}{d y}\right)^{2}
$$

$\rho$ - density of moving medium $\left(\mathrm{kg} / \mathrm{m}^{3}\right)$.

$l$ - certain reference length $(\mathrm{m})$.

$u$ - longitudinal velocity $(\mathrm{m} / \mathrm{s})$.

$y$ - transverse coordinate $(\mathrm{m})$.

The reduced differential equation is unclosed as, apart from the longitudinal velocity $u$, it contains two more unknown values - stress $\tau$ and linear dimension $l$.

Accordingly, to solve it relative to the velocity $u$, two more relationships connecting $\tau$ and $l$ values with the transverse coordinate $y$ are required.

L. Prandtl [4] proposed integrating eqn (1) at the constant transverse value of the shear stress $\tau$, which is equal to this stress on the streamlined surface $\tau_{w}\left(\tau=\tau_{w}=\right.$ const $)$, and taking the linear dimension $l$ proportional to the transverse coordinate $y$. In other words, $l=\varkappa y$, where $\varkappa$ represents the proportion factor.

Under the conditions specified previously, the following elementary differential equation is to be integrated:

$$
\sqrt{\frac{\tau_{w}}{\rho}}=\varkappa y \frac{d u}{d y}=\text { consts }
$$

Previously specified conditions predetermined the law of variation of the transverse velocity gradient. The next equation follows on from relationship (2):

$$
\frac{d u}{d y}=\frac{\text { const }}{\varkappa y}=\frac{v_{*}}{\varkappa y}
$$

where, $v_{*}=\sqrt{\frac{\tau_{w}}{\rho}}-$ dynamic velocity $(\mathrm{m} / \mathrm{s})$.

In other words, the hyperbolic law of the transverse velocity gradient variation in the turbulent boundary layer and the resultant logarithmic law of the velocity variation in the transverse section of the mentioned layer were laid on the basis of intuitive considerations. 
Equation (3) is fulfilled neither near the wall, where its right part is increasing with no limits, nor on the outer limit of the boundary layer, where its left part is going to zero.

In this connection, it is important to mention that the condition $\tau(y)=$ const and the linear relationship of the displacement length $l$ from the transverse coordinate $y$ were taken initially specifically for the near-wall region, where the differential eqn (3) is not fulfilled. Formally, the integration of this equation results in the known logarithmic velocity profile:

$$
\frac{u}{v_{*}}=\frac{1}{\varkappa} \ln y+C
$$

The obtained formula should meet the basic boundary condition resulting from the adhesion theory: at $y=0$ and $u=0$ (in this case at $y=0$ and $u \rightarrow \infty$ ). Also, this relationship does not fulfill the shear stress finiteness on the wall $\tau_{w}$, as at $y=0 \frac{\partial u}{\partial y} \rightarrow 0$.

In addition, a boundary condition is not fulfilled on the outer limit of the boundary layer where, at $y=\delta$ ( $\delta$ represents the thickness of the boundary layer), $\frac{d u}{d y} \neq 0$.

The above-mentioned circumstance is a natural consequence of the impossibility of providing equality of left and right equation parts (3) in specified regions.

In other words, the obtained velocity profile cannot be used in the most important flow region determining the interaction of the moving environment with streamlined surfaces.

There are only two options in a situation like this. Either the solution needs to be considered to be wrong, which is natural as all the above-accepted assumptions contradict the real physics, or the flow pattern itself needs to be corrected to Formula (4). Paradoxically, further development of the turbulent boundary layer theory has opted for the second option - correcting the flow pattern in the near-wall region.

The essence of this correction comes down to adoption of the double-layer - and in some cases three-layer - structural model of the turbulent boundary layer.

According to this model, the turbulent boundary layer is considered as a combination of the laminar sublayer contiguous to the streamlined surface, where the turbulent tangential stresses are excluded (no velocity and pressure fluctuations), and the value $\tau=\tau_{\text {lam }}$ is determined only by the molecular viscosity of the fluid and the main turbulent part where the velocity profile is described by eqn (4).

The known logarithmic velocity profile was obtained as a result of binding the velocity in the lower part of the turbulent boundary layer with the velocity in the outer part of the laminar sublayer, which is described by the following equation:

$$
\frac{u}{v_{*}}=\frac{1}{\varkappa} \ln \frac{y v_{*}}{v}+B=2.5 \ln \frac{y v_{*}}{v}+5.5
$$

\section{COMPARISON OF EXPERIMENTAL DATA RESULTS WITH LOGARITHMIC PROFILE DATA}

Figure 1 illustrates a degree of conformity of Formula (5) to the experimental data, where the calculation relationship is associated with the experiments of Nikuradze at different values of the Reynolds numbers [5].

The good coincidence of the experimental points with calculations in the main velocity profile part and the serious deviation of experimental data from calculations near the pipe 


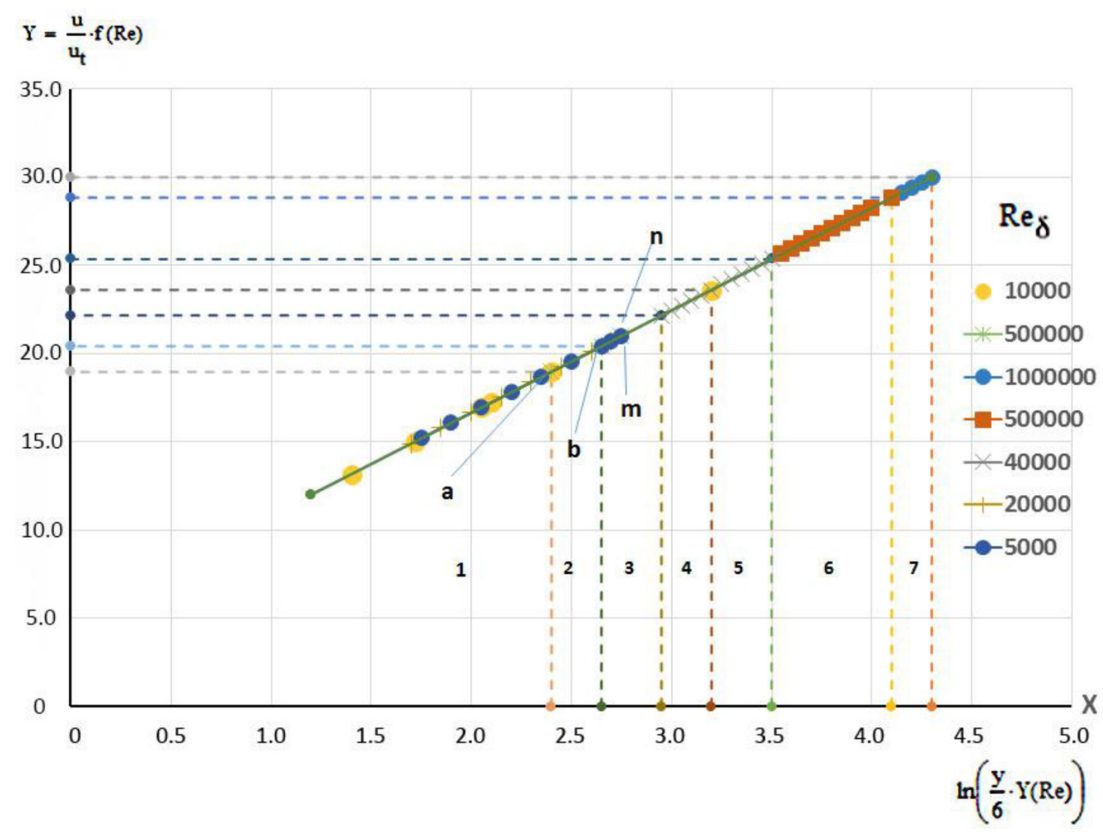

Figure 1: Experimental data on distribution of the averaged velocities in circular pipes.

walls are clearly seen. However, in coordinates taken in Fig. $1\left(\frac{u}{v_{*}}, \ln \frac{y v_{*}}{v}\right)$, the velocity profile does not depend on the Reynolds value. In that connection, Ref. [6] notes: 'The fact that the logarithmic formula retains its form for all Reynolds numbers of the flow or, in the popular phrase, is universal, is most important. From physics, the mentioned characteristic of logarithmic formulas is caused by the presence of a laminar sublayer in which the whole viscosity effect is concentrated'. Similar assessments of the logarithmic velocity profile are contained in practically all known publications [7-9].

As a result, it seems useful to analyze more precisely the mentioned fact of the universality of the logarithmic velocity profile and the degree of justification of assumptions about the presence of the laminar sublayer in the turbulent boundary layer.

To analyze the results of the comparison of the logarithmic velocity profile with the experimental data, let us represent Formula (5) as follows:

$$
\frac{u}{u_{t}} \cdot \frac{u_{t}}{v_{*}}=\frac{1}{\varkappa} \ln \frac{y}{\delta} \cdot s \frac{\delta v_{*}}{v}+B
$$

where $u_{t}$ - longitudinal velocity on the outer limit of the boundary layer $(\mathrm{m} / \mathrm{s}), \delta$ - its thickness $(\mathrm{m})$.

Due to the fact that the shear stress $\tau_{w}$ is inversely proportional to the number $\operatorname{Re}_{\delta}$ $\left(\tau_{w} \sim \frac{1}{\operatorname{Re}_{\delta}^{m}}\right)$, the following relationships are valid: $v_{*}=\sqrt{\frac{\tau_{w}}{\rho}} \sim \frac{1}{\operatorname{Re}_{\delta}^{m / 2}}$ and $\frac{u_{t}}{v_{*}} \sim \operatorname{Re}_{\delta}^{m / 2}$. 
Then, taking into account the given considerations, let us write Formula (6) as follows:

$$
\frac{u}{u_{t}} \cdot \varphi\left(R e_{\delta}\right)=\frac{1}{\varkappa} \ln \left(\frac{y}{\delta} \psi\left(R e_{\delta}\right)\right)+B
$$

This indicates that in Fig. 1 the 'floating' coordinates, maximum values of which change depending on the Reynolds value, are used instead of the fixed coordinates.

For circular pipes $\delta=r_{0}\left(r_{0}-\right.$ pipe radius) eqn (7) takes the following form:

$$
\frac{u}{u_{t}} \cdot \varphi\left(R e_{r_{0}}\right)=\frac{1}{\varkappa} \ln \left(\frac{r_{0}-r}{r_{0}} \psi\left(R e_{r_{0}}\right)\right)+B
$$

From Formula (8) it follows that for each value $R e_{r_{0}}$ the maximum value of 'floating' coordinates, in which the particular velocity profile is drawn, will change, depending on number $R e_{r_{0}}$.

In other words, $\left(\frac{u}{v_{*}}\right)_{\max }=\varphi\left(R e_{r_{0}}\right)$ and $\ln \left(\frac{r_{0} v_{*}}{v}\right)_{\text {max }}=\ln \left(\psi\left(R e_{r_{0}}\right)\right)$, as on the outer boundary layer limit (in this case in the pipe center) $\frac{y}{\delta}=\frac{r_{0}-r}{r_{0}}=1$.

While using these 'universal' coordinates, an increase in the Reynolds number increases that part of the plane $\frac{u}{v_{*}}-\ln \frac{v_{*} y}{v}$ in which experimental points of profiles are located in Fig. 1, employed in that figure to provide a comparison with the computational relationship (5).

For the purpose of greater visibility, the quadrants corresponding to these profiles are shown in dashed lines in Fig. 1.

In the 'floating' coordinates concerned, the first profile is located in the first quadrant and is determined by the straight-line segment $0-\mathrm{a}$. The second profile occupies the second quadrant and is determined by the straight-line segment $0-$ b, etc. In other words, in the reduced coordinate system, with an increase in number Re, the velocity profiles given in the mentioned dimensionless coordinates $\left(\frac{u}{v_{*}}-\ln \frac{v_{*} y}{v}\right)$ stretch along the axis of abscise in their
overlapping.

In this case, it is basically not possible to identify the impact of the factors of interest (in such a case numbers Re) on the examined velocity profiles. Thus, if in Fig. 1 the points ' $m$ ' and 'n' related, accordingly, to third (p.'m') and fourth (p.'n') profiles, are in line then it should not be inferred from this that they are 'universal', as, in the fixed coordinate system $\left(\frac{u}{u_{t}}-\frac{y}{\delta}\right)$, they are in general referred to as different values of the aforementioned dimensionless coordinates.

The 'universality' of the logarithmic velocity profile mentioned in scientific literature is a result of a more general characteristic: when an argument itself is not used as an argument but its function, the arrangement of experimental and calculation data along the line defined by two experimental points, is initially assured. 
In the case where the variation limits of the examined value and an argument determining this value are the same, then all experimental points will be arranged on the bisectrix of a right angle.

For example, if the velocity profile series are described by the power function $\frac{u}{u_{t}}=\left(\frac{y}{\delta}\right)^{n}$, where $\mathrm{n}$ is the index depending on the profile type and several criteria parameters of the Mach and Reynolds numbers type, then the experimental points for all velocity profiles will be arranged on the same line on the plane $\bar{u}-\bar{y}^{n}$, and only one point would be sufficient to find the power index $\mathrm{n}$ for each profile.

This shows that the information value of the examined experimental data presentation is extremely low, and it is difficult to draw general conclusions on that basis.

\section{CONCLUSIONS}

According to the above research, we can draw the following conclusions:

1. The accepted additional conditions and relationships, which integrate the differential equation of L. Prandtl, associating the turbulent stresses in the boundary layer with the transverse velocity gradient, are fulfilled only in the near-wall region, where the mentioned equation loses its meaning, and are inconsistent with the physical meaning in the main part of the integration.

2. This article shows that the 'universality' of the 'logarithmic' velocity profile, consisting in its independence from the Reynolds number, is a result of the use of 'floating' coordinates, the maximum value of which varies depending on the mentioned parameter.

As a result of this, an informational value of the comparison of experimental data with calculated values is eliminated, as the coincident points on the assumption curve, obtained at different values of the Reynolds numbers, refer to the different sections of the boundary layer.

3. The employed concept regarding the presence of a laminar sublayer between the streamlined surface and the turbulent part of the boundary layer is the way to give a physical meaning to the logarithmic velocity profile and does not match the interaction physics of moving media with streamlined surfaces, as the additional questions arising from that do not have physically reasoned responses.

\section{ACKNOWLEDGEMENTS}

This study, conducted by the National Research University, 'Moscow Power Engineering Institute', has been sponsored financially by the Russian Science Foundation under Agreement for Research in Pure Sciences and Prediscovery Scientific Studies No. 14-19-00944 dated July 16, 2014.

\section{REFERENCES}

[1] Keller, L.V. \& Friedman, A.A., Differential equations of turbulent motion of a compressible fluid. Proceedings of the First International Congress for Applied Mechanics, Delft (Holland), pp. 395-405, 1924.

[2] Sedov, L.I., Similarity and Dimensional Methods in Mechanics, Publishing house "Science" M, p. 440, 1972.

[3] Monin, A.S. \& Yaglom, A.M., Statistical Fluid Mechanics, Part I. Publishing house "Science" M, -p. 636, 1955. 
[4] Prandtl, L., Fluid Mechanics, Publishing house "IL". M, 1951.

[5] Schlichting, H., Boundary Layer Theory, Publishing house "Science" M. FML, M, 1969.

[6] Loitsiansky, L.G., Fluid and Gas Mechanics, Publishing house "Science" M, p. 892, 1970.

[7] Melnikov, A.P., Basics of Theoretical Mechanics, LKVVIA L. 1953.

[8] Fabrikant, N.Y., Aerodynamics. M, Publishing house "Science", 1970.

[9] Fedyaevsky, K.K., Voytkunsky, Y.I. \& Fadeev, Y.I., Fluid Mechanics, Publishing house "Shipbuilding" L, 1968. 